\title{
Review of the safety, efficacy and patient acceptability of the levonorgestrel-releasing intrauterine system
}

\author{
Chandra Kailasam' \\ David Cahill ${ }^{2}$ \\ 'Bristol Centre for Reproductive \\ Medicine, Southmead Hospital, \\ Bristol, UK; ${ }^{2}$ Dept of Obstetrics \\ and Gynaecology, University \\ of Bristol, St Michael's Hospital, \\ Bristol, UK
}

Correspondence: Chandra Kailasam Bristol Centre for Reproductive Medicine, Southmead Hospital, BSIO 9NB, UK Email: chandra.kailasam@nbt.nhs.uk

\begin{abstract}
The levonorgestrel-containing intrauterine system is an extremely effective, reversible and safe form of long-term yet reversible birth control. In view of its efficacy, it is a safer alternative to permanent contraceptive methods such as sterilization. It is especially useful in situations where use of estrogen-containing contraceptives is contraindicated. While menstrual disturbances are a common side effect, proper counseling improves compliance. In addition to its contraceptive effect, the levonorgestrel intrauterine system offers potential therapeutic benefits in other clinical contexts, including menorrhagia, symptomatic fibroids, endometriosis, and endometrial protection.
\end{abstract}

Keywords: intrauterine device, levonorgestrel intrauterine device, contraception

\section{Introduction}

The levonorgestrel-containing intrauterine device is a very effective and safe form of reversible long-term birth control. In addition to its contraceptive effect, it offers potential non-contraceptive therapeutic benefits.

We reviewed the efficacy, safety and clinical applications of the levonorgestrelcontaining intrauterine device (LNG-IUS, Mirena ${ }^{\circledR}$ ). The search included the PubMed, Cochrane Controlled Trials Register and WHO publications on contraception. We included randomized controlled trials, controlled clinical trials and systematic/clinical reviews published in the English language in peer-reviewed journals and guidelines published by the WHO and the National Institute for Clinical Excellence (NICE). The key words used to search data included IUD, IUD-IUS, contraception.

\section{Rationale for IUD}

Among the several long-acting contraceptive methods, the intrauterine device (IUD) is the most popular and overall it is second most popular contraceptive method worldwide after sterilization (Progress in Reproductive Health Research 2002). The popularity of the IUD stems from the fact that in addition to providing long-lasting, highly effective, rapidly reversible contraception, it has no known effects on breast milk or breastfeeding; it does not interfere with sexual intercourse or with any type of medication; it is widely available throughout the world, it can used by women of any age or parity and following an abortion or miscarriage and finally once in place, its user can more or less forget about it with no further costs (WHO 2007). There is no evidence that use of IUD increases tubal infertility (Grimes 2000). 
In addition to its contraceptive effect, the LNG-IUS offers potential therapeutic benefits in other clinical contexts, including menorrhagia, symptomatic fibroids, endometriosis, and endometrial suppression.

\section{Development and pharmacology of Mirena ${ }^{\circledR}$ intrauterine contraceptive device}

The aim of progesterone-releasing intrauterine systems initially was to reduce IUCD expulsion, by the addition of 'uterine relaxing hormones' (Odlind 1996). This led to the development of Progestasert ${ }^{\circledR}$ (the first hormonally impregnated device releasing $65 \mu \mathrm{g}$ of progesterone per day) and Mirena ${ }^{\circledR}$ LNG-IUS (releasing $20 \mu \mathrm{g}$ of levonorgestrel per day).

Mirena ${ }^{\circledR}$ LNG-IUS was developed by Leiras Oy, Turku, Finland, and was launched first in Finland in 1990. It has a T-shaped body $(32 \times 32 \mathrm{~mm})$ made of polyethylene with an elastomer sleeve consisting a 1 to 1 mixture of polydimethylsiloxane and $52 \mathrm{mg}$ of levonorgestrel mounted around its vertical part. The sleeve is covered with a drug-releasecontrolling membrane of medical grade polydimethylsiloxane that releases levonorgestrel over an extended time of up to 5 years at a practically constant rate. The initial release rate of levonorgestrel is $20 \mu \mathrm{g}$ per $24 \mathrm{~h}$, and at the end of 5 years the release rate is still above $10 \mu \mathrm{g}$ per $24 \mathrm{~h}$ (Lähteenmäki et al 2000). The distal end of the T-frame contains 2 removal threads. The device also contains barium sulfate, which makes it visible on X-ray examination. Local delivery of LNG results in low but detectable serum levels of LNG (0.1-0.4 ng/mL), much lower than peak levels observed with other combined or progestin-only contraceptives containing levonorgestrel (ESHRE Workshop 2008).

Levonorgestrel is a highly effective progestin, with an estimated progestational potency 10 times greater than that of progesterone; it also exhibits some androgenic properties (Sitruk-Ware 2007). The recommended duration of use of the Mirena ${ }^{\circledR}$ coil is 5 years. Even though the licensed duration of action is 5 years, evidence suggests that it is effective as a contraceptive for up to 7 years (Sivin et al 1991). Furthermore, women who are aged 45 years or older when their LNG-IUS is inserted and are amenorrhoeic may keep it until they no longer need contraception, even if this is beyond the duration of UK Marketing Authorisation (NICE guidelines 2005).

Mirena ${ }^{\circledR}$ LNG-IUS is currently licensed in the UK as a 5-year contraceptive agent (license awarded 1995), treatment for idiopathic menorrhagia (license awarded 2001), and to provide uterine protection during estrogen replacement therapy in perimenopausal and postmenopausal women (license awarded 2005). The second and third applications for Mirena ${ }^{\circledR}$ LNG-IUS are not licensed in the US or Canada (Varma et al 2006).

\section{Mechanism of action}

The LNG-IUS acts predominantly by preventing implantation and sometimes by preventing fertilization. The contraceptive effects of the LNG-IUS are mediated via its progestogenic effect on the endometrium. Local intrauterine delivery of levonorgestrel (LNG) results in extensive decidualization of endometrial stromal cells, atrophy of the glandular and surface epithelium, and changes in vascular morphology (suppression of spiral artery formation and presence of large dilated vessels) along with down-regulation of sex steroid receptors in all cellular components (Guttinger and Critchley 2007). The result is a thin decidualized endometrium, an environment that is unsuitable for sperm survival, fertilization and implantation. The endometrial changes develop in the first month after insertion and persist until the device is removed (Guttinger and Critchley 2007). By inactivating the endometrium and suppressing proliferation, it also decreases menstrual blood loss (MBL) and pain. The levonorgestrel released locally alters the quality of cervical mucus, making it hostile to the movement of sperm through the cervix (Jonsson et al 1991). Thus, the number and quality of sperm reaching the site of fertilization in the tube seems to be reduced in LNG-IUS users.

Ovulation is not suppressed as it has little influence on ovarian activity; women have normal estradiol values from the time of insertion through its 5-year life span (Luukkainen et al 1990) ensuring that the LNG-IUS would not expose the user to hypoestrogenism leading to osteoporosis (Bahamondes et al 2006). Although the anovulation rate is almost $85 \%$ at the beginning of use, this rate falls to less than $15 \%$ at the end of the first year (Nilsson et al 1980). As ovulatory cycles occur in most, even amenorrheic, users, ovulation suppression is not the primary mode of action (Lähteenmäki et al 2000). Serum levels of LNG are usually not sufficient to suppress ovulation, as a release of $50 \mu \mathrm{g}$ per $24 \mathrm{~h}$ of LNG would be necessary to completely inhibit ovulation (Lähteenmäki et al 2000). The local progestative effect of the LNG-IUS on the endometrium manifests within a period of 3 months and over after insertion (Zalel et al 2003). This means that it can take up to 3 months for the initial menstrual disturbances to settle. Women should be accordingly counseled so as to 
decrease the discontinuation rate of the LNG-IUS due to the initial menstrual disturbances.

\section{Insertion}

The LNG-IUS can be inserted at any time in the menstrual cycle if it is reasonably certain the woman is not pregnant. However compared with the $\mathrm{Cu}$-IUD that is effective immediately, it takes 7 days to provide effective contraceptive protection. Hence additional contraception or abstinence should be advised for 7 days after inserting the LNG-IUS unless inserted in the first 7 days of the cycle or when switching from a different method of contraception unless the current contraceptive method is still effective (FSRH Guidance 2007). While the insertion procedure may be relatively easy compared with insertion of other IUDs, some women may need analgesia and cervical dilatation (Jensen et al 2008). IUDs can be inserted immediately after first or second trimester abortion and from 4 weeks post partum, irrespective of the mode of delivery (El Tagy 2003; NICE 2005). In complicated valvular heart disease, prophylactic antibiotics should be used at the time of insertion to prevent endocarditis (WHO 2004).

\section{Contraindications}

The LNG-IUS should be avoided in patients with unexplained vaginal bleeding. It is preferably avoided in the presence of sexually transmitted diseases such as chlamydia and gonorrhea. In a systematic review, Mohllajee et al (2006) reported that with IUD insertion in the presence of chlamydia infection or gonorrhea, subsequent pelvic inflammatory disease (PID) rates were $0 \%-5 \%$, compared with insertion in the absence of infection $(0 \%-2 \%)$.

\section{Therapeutic benefits of the LNG-IUS}

\section{Contraceptive benefits}

The LNG-IUS provides highly effective contraception and is equally efficient in all age groups with the risk of failure similar throughout the life span of the device. The 5-year cumulative pregnancy rate per 100 users is 0.5 and the 5 -year Pearl rate 0.11 (Backman et al 2004). The cumulative pregnancy rate at 5 years is $<0.5 \%$ (Thonneau and Almont 2008). Its use in lactating women provides highly effective and acceptable contraception and does not negatively influence breast-feeding or the growth and development of breast-fed infants (Shaamash et al 2005). Women with an intrauterine pregnancy with an LNG-IUS in situ should be advised to have the LNG-IUS removed before 12 completed weeks' gestation whether or not they intend to continue the pregnancy (NICE 2005).

It can be safely used in women with a past history of PID or ectopic pregnancy, women with fibroids, and in young nulliparous women (WHO 2004). The LNG-IUS is medically safe for women to use if oestrogen is contraindicated (NICE 2005).

The LNG-IUS is both safe and extremely efficacious for use in nulliparous women with no greater risk of perforation or expulsion (Prager and Darney 2007). In fact the LNG may be protective against infection via thickening of the cervical mucus (Jonsson et al 1991) and decreased menstrual blood loss. Nulliparous users are at no increased risk for infection and infertility than multiparous users and it is safe to offer post-abortion placement of the LNG-IUS to nulliparous women (Prager and Darney 2007). In a randomized study of young nulliparous women (Suhonen et al 2004), the safety and acceptability of the LNG-IUS for contraception was observed to be as good as with oral contraceptives, with a high continuation rate. The discontinuation rate in the first year of the LNG-IUS is $20 \%$, indicating that acceptability is similar among nulliparous and parous women (Prager and Darney 2007).

In contrast to the copper IUDs, the LNG-IUS is not recommended for emergency contraception. The absence of embryotoxic copper ions and the relatively low serum levels of levonorgestrel obtained immediately following LNG-IUS insertion compared with standard hormonal emergency contraception suggests that it may not be effective (ESHRE Capri Workshop Group 2008).

The LNG-IUS has been favorably compared with other contraceptive methods. In randomized comparative trials (RCTs), pregnancy rates were significantly lower with the LNG-IUS than with copper devices (Sivin et al 1991; Andersson et al 1994; Pakarinen et al 2003), though a Cochrane review of 21 RCTs (French et al 2004) concluded that there is insufficient evidence to conclude that the LNG-IUS is more effective than copper IUDs. In a recent systematic review of the Cochrane Library for all IUD-related reviews (Grimes et al 2007), the LNG-IUS was found to have comparable efficacy to that of IUDs with $>250 \mathrm{~mm}^{2}$ of copper, immediate post-partum, and post-abortal insertion appeared safe and effective and prophylactic antibiotics at the time of insertion appeared unwarranted except in populations with a high prevalence of sexually transmitted infections (STIs). The LNG-IUS and tubal sterilization have comparable high effectiveness, with the LNG-IUS a safer option, and all women, particularly young women, who are 
at high risk for sterilization regret, should be encouraged to consider the LNG-IUS in place of a surgical procedure that is potentially irreversible (Grimes and Mishell 2008).

Repeated use of the device has had favorable outcomes. The initial bleeding problems that are frequently observed after the insertion of the first LNG-IUS do not recur after an immediate change from the first IUS to the second IUS (Rönnerdag and Odlind 1999). In contrast to other long acting progestin only contraceptives, LNG-IUS has no effect on bone mineral density (Inki et al 2007).

There does not seem to be a delay in the return of fertility following removal of the Mirena ${ }^{\circledR}$ coil with conception rates 79.1/100 women at 12 months after removal (Andersson et al 1992).

\section{Non-contraceptive benefits of the LNG-IUS}

\section{Menorrhagia}

Available medical treatments for menorrhagia include the LNG-IUS, non-steroidal anti-inflammatory drugs, antifibrinolytic drugs, progestogens, oral contraceptives, and danazol. The choice of medical treatment can depend on individual factors such as requirement for contraceptive and dysmenorrhea. However, first-line therapy with drugs has variable efficacy and, at best, oral medication reduces menstrual blood loss by only 50\% (Istre and Qvigstad 2007). The immediate and intense suppression of the endometrium leads to over $90 \%$ reduction of menstrual blood loss over a period of 12 months (Anderson and Rybo 1990) along with significant beneficial increase in hemoglobin and ferritin levels (Xiao et al 2003).

In a randomized controlled trial (Hurskainen et al 2004) health-related quality-of-life outcomes associated with the LNG-IUS and hysterectomy was similar with financial benefits in favor of the LNG-IUS. In a Cochrane review of 10 randomized control trials (Lethaby et al 2005), the LNG-IUS was more effective than other medical interventions, with a $90 \%$ reduction from baseline in menstrual blood loss. Although the LNG-IUS results in a smaller reduction in menstrual blood loss than endometrial ablation, there are no differences in the women's rates of satisfaction or quality of life. In a Cochrane systematic review of 8 trials (Marjoribanks et al 2006), use of LNG-IUS was more cost effective, with levels of satisfaction and quality of life with an LNG-IUS system similar to those after surgical treatment such as transcervical endometrial resection or balloon ablation or hysterectomy. Further longterm studies are needed to compare the effectiveness of the LNG-IUS against conservative surgical treatments.
Inherited bleeding disorders may be the cause of menorrhagia in up to $13 \%$ of women and the LNG-IUS is an effective treatment option in such women (Kadir and Chi 2007), as medical treatments may otherwise be contraindicated and surgery carries additional risks. It is also an effective treatment for menorrhagia in women receiving oral anticoagulation (Pisoni 2005).

The LNG-IUS is cost effective in the treatment of menorrhagia, while offering reliable contraception. Compared with oral contraceptives and surgical treatment, treatment strategies employing the LNG-IUS are the most cost-effective in managing dysfunctional uterine bleeding in women not desiring additional children (Blumenthal et al 2006). LNG-IUS followed by endometrial ablation may be the most cost-effective treatment for menorrhagia, when compared with immediate surgery (Clegg et al 2007).

\section{Endometriosis}

The LNG-IUS delivers significant amounts of levonorgestrel into the peritoneal fluid (Lockhat et al 2005) and this may explain the pain relief in patients with peritoneal endometriosis.

Medical treatments that are based on the reduction of lesions or on ovarian estrogen suppression, cause profound hypoestrogenism inducing a decrease in bone mineral density and hence treatment is limited 6 months (d'Arcangues 2006), although longer treatment with add-back hormone therapy is possible. In addition, there are systemic side effects, and the need for regular administration could affect compliance. In such patients the LNG-IUS can be a useful alternative.

In a systematic review on the use of LNG-IUS for symptomatic endometriosis following surgery, post-operative use of the LNG-IUS reduced the recurrence of painful periods in women who have had surgery for endometriosis while there was insufficient evidence for other benefits such as reduced likelihood of further surgery for endometriosis and improved long-term fertility (Abou-Setta et al 2006). In a randomized controlled trial, insertion of the Mirena ${ }^{\circledR}$ coil significantly reduced the medium-term risk of recurrence of moderate or severe dysmenorrhea compared with expectant management following operative laparoscopy for symptomatic endometriosis (Vercellini et al 2003). In another RCT (Petta et al 2005), LNG-IUS and depot-GnRH-analog were equally effective in significantly decreasing endometriosisrelated pain. However an advantage with LNG-IUS is the fact that it does not provoke hypoestrogenism while being effective for 5 years. There is insufficient information on 
the efficacy of the LNG-IUS in the possible prevention of endometriosis recurrence.

\section{Adenomyosis}

The LNG-IUS has been reported to be useful in women with adenomyosis, although studies have been limited by small numbers. Its use may significantly reduce pain and abnormal bleeding associated with adenomyosis along with significantly reduced adenomyotic lesions, as evaluated by the thickness of the junctional zone (Braghetoa et al 2007). A long-term study showed that the use of the LNG-IUS led to significant pain relief, reduction in the uterine volume and menstrual blood loss volume, and improvements in hematologic indices in patients with adenomyosis (Cho et al 2008); however, there was a gradual increase in uterine volume, pain scores, and pictorial blood loss assessment chart scores at 2 years after insertion and the authors suggested that to maintain the efficacy of the LNG-IUS for the management of adenomyosis, a new device might be needed after 3 years.

\section{Fibroids}

The LNG-IUS appears safe and effective in the treatment of menorrhagia in women with uterine cavities distorted by submucosal fibroids (Soysal and Soysal 2005). A recent review (Kaunitz 2007) of the published literature suggested that, in women with uterine fibroids, with or without menorrhagia, the LNG-IUS reduces menstrual blood loss and likely reduces menstrual pain while maintaining high contraceptive efficacy. However, expulsion rates are higher and there is inconsistent evidence on whether the LNG-IUS decreases uterine/fibroid dimensions. Although symptomatic improvement may not be uniform, these findings indicate that the LNG-IUS is a useful therapeutic option for selected women with menstrual symptoms associated with uterine fibroids.

\section{Endometrial protection}

The targeted delivery of progestagen in the uterine cavity is a preferred route in women who need endometrial protection due to the absence of systemic side effects along with high efficacy.

Use of oral tamoxifen as adjuvant therapy for women with breast cancer has improved survival rates. However, it exerts weak estrogenic effect on the endometrium and hence is associated with endometrial pathologies such as polyps, hyperplasia and endometrial cancer. In view of its progestational effects, the LNG-IUS is an effective prophylaxis in the prevention of endometrial pathology in women receiving tamoxifen (Chan et al 2007; Gardner et al 2000).

The LNG-IUS adequately suppresses the endometrium during hormone replacement therapy with estrogens (Riphagen et al 2000) while avoiding the potential adverse systemic effects of progestogens. A literature review by Riphagen et al (2000) and a subsequent long-term study of post-menopausal women by Wildemeersch et al (2007a) highlighted the endometrial protection offered by the LNG-IUS in women receiving estrogen replacement therapy.

The LNG-IUS has been investigated in the treatment of non-atypical and atypical hyperplasia as a useful alternative to hysterectomy especially in younger women who still wish to become pregnant or in women who refuse operation or are in poor health. While studies (Wildemeersch et al 2007b; Varma et al 2008) suggest it may be an effective option for suppressing the endometrium, there have been reports of progression of atypical endometrial hyperplasia to adenocarcinoma despite intrauterine progesterone treatment with the levonorgestrel-releasing intrauterine system (Kresowick et al 2008). Hence extreme caution should be exercised and we need robust randomized controlled trials to evaluate the effectiveness of the LNG-IUS in treating endometrial hyperplasia.

\section{Side effects}

The adverse events of interest fall into 2 categories: those related to an intrauterine device, such as dysmenorrhea, irregular bleeding, ectopic pregnancy, and expulsion of the device; and those related to progestogens, such as bloating, weight gain, and breast tenderness. In a systematic review of the literature, reported cumulative discontinuation rates with the LNG-IUS were as high as $24 \%$ after 1 year and $33 \%$ after 2 years (NICE 2005).

\section{Bleeding complications}

Overall, the commonest reason for discontinuation is unacceptable bleeding patterns.

Up to $60 \%$ of women stop using the LNG-IUS within 5 years, which is similar to other IUDs, unacceptable vaginal bleeding and pain being the most common reasons for discontinuation (NICE 2005). Even though irregular bleeding and spotting are common during the first 6 months following LNG-IUS insertion, oligomenorrhea or amenorrhea is likely by the end of the first year of LNG-IUS use (NICE 2005). Since frequent irregular bleeding is common during the first few months following system insertion, proper counseling of the patient about possible bleeding patterns is crucial in 
order to minimize premature LNG-IUS removals. Since amenorrhea is an expected outcome (occurring in about $20 \%$ of users at 12 months), adequate counseling provides reassurance that the absence of bleeding does not generally signify pregnancy or other problems leading to high continuation rate and high level of patient satisfaction (Jensen et al 2008).

Information received at the insertion visit is strongly associated with increased user satisfaction among the users of the LNG-IUS (Backman et al 2002), the association between high user satisfaction and advance information being strongest on the possibility of missing periods.

\section{Uterine perforations}

Incidence of uterine perforations related to the insertion of a LNG-IUS is around 2.6 per 1000 insertions (Van Houdenhoven et al 2006). Insertion in lactating women, even beyond 6 weeks after delivery, is an important risk factor. The manufacturer of the LNG-IUS currently recommends that post-partum insertions should be postponed until 8 weeks after delivery. Uterine perforation at insertion seems less likely to occur if a withdrawal rather than a push-out technique - the recommended technique for a LNG-IUS - is used.

\section{Expulsion and displacement}

Expulsion of an IUD occurs in approximately 1 in 20 women, and is most common in the first 3 months after insertion (NICE 2005). Patients at increased risk of expulsion include nulliparous women, women with severe dysmenorrhea, and those with insertions immediately post partum or post abortion. There is insufficient evidence to indicate that expulsion rates are lower with LNG-IUS (Chrisman et al 2007). There are no differences in the rates of expulsion between $\mathrm{Cu}$-IUDs and the LNG-IUS (FSRH 2007). As expulsion generally occurs within the first few months, women should be encouraged to attend follow-up within 12 weeks of insertion.

It is rare for the LNG-IUS to get displaced and there is conflicting evidence on how best to manage these patients. Intra-peritoneal dislocated LNG-IUS results in plasma levonorgestrel levels 10 times higher $(4.7 \mathrm{nmol} / \mathrm{L})$ than those seen with LNG-IUS placed in utero. This high plasma levonorgestrel level suppresses ovulation and therefore it has been suggested that a misplaced LNG-IUS should be removed when pregnancy is desired, as opposed to the copper IUD that may be left intraperitoneally, especially if asymptomatic (Haimov-Kochman et al 2003). However pregnancies have also been documented with a displaced LNG-IUS (Budiman et al 2007).

\section{Ectopic pregnancy}

The LNG-IUS is a very effective contraceptive and the absolute risk of pregnancy (intrauterine and ectopic) is very low. A previous ectopic pregnancy is not a contraindication to the use of intrauterine contraception (FSRH 2007). The risk of ectopic pregnancy when using the LNG-IUS is lower than when using no contraception. The overall risk of ectopic pregnancy when using the LNG-IUS is very low, at about 1 in 1000 in 5 years. If a woman becomes pregnant with the LNGIUS in situ, the risk of ectopic pregnancy is about 1 in 20 (NICE 2005). Similar rates of ectopic pregnancy are reported for the LNG-IUS and Cu-IUDs (French et al 2004).

\section{Infection}

The risk of developing PID following LNG-IUS insertion is very low (less than 1 in 100) in women who are at low risk of STIs and removals due to PID among LNG-IUS users is below $1 \%$ at 1 year, and below $1.5 \%$ at 5 years (NICE 2005). A systematic review reported that there is conflicting evidence on whether levonorgestrel IUD is associated with a lower risk of PID than other IUDs and any risk of upper-genital-tract infection after the first month is small (Grimes 2000). The protective effect of the LNG-IUS may be due to impenetrable cervical mucus, endometrial changes, or reduced retrograde menstruation (Toivonen et al 1991). If a woman was to develop PID with the IUD in place, it may be reasonable to offer initial treatment without immediate removal (WHO 2004). In rare cases of pelvic infection secondary to Actinomyces israelii, device removal in conjunction with antibiotic treatment is more successful at clearing the colonization than antibiotics alone (Bonacho et al 2001).

A woman who currently has an STI such as gonorrhea or chlamydia or is at very high risk should not have an IUD inserted as insertion may increase the risk of PID. If a high risk patient screens negative, then an IUD can be inserted and if the screen is positive, then an IUD can be inserted after treatment, if she is not at risk of reinfection by the time of insertion (WHO 2007). In exceptional circumstances, if other, more appropriate methods are not available or not acceptable, an IUD can be inserted in high risk individuals even if STI testing is not available. Presumptive treatment should be considered with a full curative dose of antibiotics effective against both gonorrhea and chlamydia and inserting the IUD after completion of treatment. The patient should be carefully checked for signs of infection at followup and treated accordingly while being advised to return at once if there are any signs of infection (WHO 2007). 
Farley et al (1992) reported that PID among IUD users is most strongly related to the background risk of STI and hence screening for chlamydia should always be considered prior to inserting the LNG-IUS.

A systematic review to assess the effectiveness of prophylactic antibiotic administration before IUD insertion in reducing IUD-related complications and discontinuations within 3 months of insertion highlighted the low risk of IUD-associated infection, with or without use of antibiotic prophylaxis (Grimes and Schulz 2001). Another systematic review (Mohllajee et al 2006) suggested that women with chlamydial infection or gonorrhea at the time of IUD insertion were at increased risk of PID relative to women without infection, the absolute risk of PID being low for both groups. However, whether IUDs increase the risk of PID in women with an STI at the time of insertion is not known (Mohllajee et al 2006).

\section{Ovarian cysts}

The use of the LNG-IUS is associated with a small risk of development of ovarian cysts (Inki et al 2002). The precise mechanism by which the ovarian cysts are caused is not known, but may be secondary to disturbances in the normal growth and rupture of follicles during LNG-IUS use. However in a prospective, randomized trial by Inki et al (2002) these were symptomless and showed a high rate (94\%) of spontaneous resolution and hence no routine ultrasound screening is necessary of women using the LNG-IUS.

\section{Other rare side effects}

Unrecognized retention in the uterine cavity of the active part (hormone-releasing capsule) of an LNG-IUS may lead to secondary amenorrhea. Although LNG-IUS are inserted and removed without particular difficulty in most cases, it may be prudent to check the device following removal to ensure that the capsule remains attached to the rest of the device (Forrest et al 2008).

\section{Hormonal complications}

The systemic absorption of levonorgestrel may have the potential to cause hormonal side effects. The LNG-IUS releases $20 \mu \mathrm{g}$ per day of levonorgestrel and so drugrelated adverse events are less frequent than with the oral preparations of progesterone, which result in higher serum concentrations.

However discontinuation due to hormonal (non-bleeding) problems is rare. While changes in mood and libido or weight gain are similar whether using the LNG-IUS or IUDs, there is an increased possibility of developing acne (NICE 2005). While some women may complain of headaches, women who have migraine with or without aura may use the LNG-IUS.

The use of the LNG-IUS has not been associated with an increased risk of breast cancer (Backman et al 2005). In women with a past history of breast cancer, Trinh et al (2007), reported that, overall, there was no increased risk of breast cancer recurrence associated with use of the LNG-IUS; subgroup analysis suggested that while the LNG-IUS is not associated with an increased risk of recurrence in patients who start using the LNG-IUS after completing their breast cancer treatment, women who developed breast cancer while using an LNG-IUS and who continued to use the LNG-IUS, showed a higher risk of recurrence of borderline statistical significance. Hence additional research is needed to confirm or refute these findings (Trinh et al 2007).

\section{Conclusion}

Women contemplating undergoing sterilization or hysterectomy seek a long-term solution for contraception or treatment of menorrhagia. The LNG-IUS is one of the most versatile forms of long-acting reversible method of contraception. New developments in the delivery of levonorgestrelreleasing intrauterine devices such as the Femilisk ${ }^{\circledR}$ (parous women), the Femilisk Slim ${ }^{\circledR}$ (nulliparous women), and the frameless FibroPlant ${ }^{\circledR}$ levonorgestrel LNG-IUS possess features that may solve the main problems encountered with conventional IUDs (eg, expulsion, abnormal or excessive bleeding, and pain) (Wildemeersch 2007). The LNG-IUS system is an extremely effective contraceptive and has many non-contraceptive health benefits, including suppression of menstruation, maintenance of iron stores, improvement in dysmenorrheal, and endometrial protection for women on estrogen replacement therapy.

\section{Disclosures}

Neither author has any conflicts of interest to disclose.

\section{References}

Abou-Setta AM, Al-Inany HG, Farquhar CM. 2006. Levonorgestrelreleasing intrauterine device (LNG-IUD) for symptomatic endometriosis following surgery. Cochrane Database of Systematic Reviews, Issue 4.

Andersson K, Batar I, Rybo G. 1992. Return to fertility after removal of a levonorgestrel-releasing intrauterine device and Nova-T. Contraception, 46:575-84.

Andersson K, Odlind V, Rybo G. 1994. Levonorgestrel-releasing and copper-releasing (Nova T) IUDs during five years of use. A randomized comparative trial. Contraception, 49:56-72.

Anderson JK, Rybo G. 1990. Levonorgestrel-releasing intrauterine device in the treatment of menorrhagia. Br J Obstet Gynaecol, 97:690-4 
Backman T, Huhtala S, Luoto R, et al. 2002. Advance information improves user satisfaction with the levonorgestrel intrauterine system. Obstet Gynecol, 99:608-13.

Backman T, Rauramo I, Huhtala S, et al. 2004. Pregnancy during the use of levonorgestrel intrauterine system. Am J Obstet Gynecol, 190:50-4.

Backman T, Rauramo I, Jaakkola K, et al. 2005. Use of the levonorgestrelreleasing intrauterine system and breast cancer. Obstet Gynecol, 106:813-7.

Bahamondes L, Espejo-Arce X, Hidalgo MM, et al. 2006. A cross-sectional study of the forearm bone density of long-term users of levonorgestrelreleasing intrauterine system. Hum Reprod, 21:1316-9.

Blumenthal PD, Trussell J, Singh RH, et al. 2006. Cost-effectiveness of treatments for dysfunctional uterine bleeding in women who need contraception. Contraception, 74:249-58.

Bonacho I, Pita S, Gomez-Besteiro MI. 2001. The importance of the removal of the intrauterine device in genital colonization by actinomyces. Gynecol Obstet Invest, 52:119-23.

Braghetoa AM, Casertab N, Bahamondes L, et al. 2007. Effectiveness of the levonorgestrel-releasing intrauterine system in the treatment of adenomyosis diagnosed and monitored by magnetic resonance imaging. Contraception, 76:195-9.

Budiman H, Kümper C, Mylonas I, et al. 2007. Pregnancy in a patient with a displaced levonorgestrel-releasing intrauterine system: a case report. Arch Gynecol Obstet, 276:263-4.

Chan S, Tam W, Yeo W, et al. 2007. A randomised controlled trial of prophylactic levonorgestrel intrauterine system in tamoxifen-treated women. BJOG, 114:1510-5.

Clegg JP, Guest JF, Hurskainen R. 2007. Cost-utility of levonorgestrel intrauterine system compared with hysterectomy and second generation endometrial ablative techniques in managing patients with menorrhagia in the UK. Curr Med Res Opin, 23:1637-48.

Cho SH, Nam A, Kim HY, et al. 2008. Clinical effects of the levonorgestrelreleasing intrauterine device in patients with adenomyosis. Am JObstet Gynecol, 198:373.e1-7.

Chrisman C, Ribeiro P, Dalton VK. 2007. The levonorgestrel-releasing intrauterine system: an updated review of the contraceptive and noncontraceptive uses. Clin Obstet Gynecol, 50:886-97.

d'Arcangues C. 2006. WHO statement on hormonal contraception and bone health. Contraception, 73:443-4.

El Tagy A, Sakr E, Sokal DC, et al. 2003. Safety and acceptability of postabortal IUD insertion and the importance of counselling. Contraception, 67:229-34.

ESHRE Capri Workshop Group. 2008. Intrauterine devices and intrauterine systems. Hum Reprod Update, 14:197-208.

Farley TMM, Rowe PJ, Meirik O, et al. 1992. Intrauterine devices and pelvic inflammatory disease: an international perspective. Lancet, 339:785-8.

Forrest A, Amarakone I, Lord J. 2008. Retained hormone release capsule following removal of Mirena intrauterine system. BJOG, 115:130-1.

French R, Van Vliet H, Cowan F, et al. 2004. Hormonally impregnated intrauterine systems (IUSs) versus other forms of reversible contraceptives as effective methods of preventing pregnancy. Cochrane Database of Systematic Reviews, Issue 3. Art. No: CD001776.

[FSRH] Faculty of Sexual and Reproductive Healthcare Guidance. Clinical Effectiveness Unit. 2007. Intrauterine Contraception.

Gardner FJ, Konje JC, Abrams KR, et al. 2000. Endometrial protection from tamoxifen-stimulated changes by a levonorgestrel-releasing intrauterine system: a randomised controlled trial. Lancet, 356:1711-7.

Grimes DA. 2000. Intrauterine device and upper-genital-tract infection. Lancet, 356:1013-109.

Grimes DA, Lopez LM, Manion C, et al. 2007. Cochrane systematic reviews of IUD trials: lessons learned. Contraception, 75:S55-S9.

Grimes D, Mishell Jr D. 2008. Intrauterine contraception as an alternative to interval tubal sterilization. Contraception, 77:6-9.

Grimes DA, Schulz KF. 2001. Antibiotic prophylaxis for intrauterine contraceptive device insertion. Cochrane Database Syst Rev, CD001327.
Guttinger A, Critchley HO. 2007. Endometrial effects of intrauterine levonorgestrel. Contraception, 75:S93-S8.

Haimov-Kochman R, Amsalem H, Adoni A, et al. 2003. Management of a perforated levonorgestrel-medicated intrauterine device - a pharmacokinetic study: case report. Hum Reprod, 18:1231-3.

Hurskainen R, Teperi J, Rissanen P. 2004. Clinical outcomes and costs with the levonorgestrel-releasing intrauterine system or hysterectomy for treatment of menorrhagia: randomized trial 5-year follow-up. JAMA, 291:1456-63

Inki P. 2007. Long-term use of the levonorgestrel-releasing intrauterine system. Contraception, 75(6 Suppl):S161-6.

Inki P, Hurskainen R, Palo P, et al. 2002. Comparison of ovarian cyst formation in women using the levonorgestrel-releasing intrauterine system vs. hysterectomy. Ultrasound Obstet Gynecol, 20:381-5.

Istre O, Qvigstad E. 2007. Current treatment options for abnormal uterine bleeding: an evidence-based approach. Best Pract Res Clin Obstet Gynaecol, 21:6:905-13.

Jensen JT, Nelson AL, Antonio C. 2008. Subject and clinician experience with the levonorgestrel-releasing intrauterine system Contraception, 77:22-9.

Jonsson B, Landgren B-M, Eneroth P. 1991. Effects of various IUDs on the composition of cervical mucus. Contraception, 43:447-58.

Kresowik J, Ryan GL, Van Voorhis BJ. 2008. Progression of atypical endometrial hyperplasia to adenocarcinoma despite intrauterine progesterone treatment with the levonorgestrel-releasing intrauterine system. Obstet Gynecol, 111:547-9.

Kadir RA, Chi C. 2007. Levonorgestrel intrauterine system: bleeding disorders and anticoagulant therapy. Contraception, 75:S123-S9.

Kaunitz AM. 2007. Progestin-releasing intrauterine systems and leiomyoma. Contraception, 75:S130-S3.

Lähteenmäki P, Rauramo I, Backman T. 2000. The levonorgestrel intrauterine system in contraception. Steroids, 65:693-7.

Lethaby AE, Cooke I, Rees M. 2005. Progesterone or progestogen-releasing intrauterine systems for heavy menstrual bleeding. Cochrane Database of Systematic Reviews, Issue 4. Art. No: CD002126

Lockhat FB, Emembolu JE, Konje JC. 2005. Serum and peritoneal fluid levels of levonorgestrel in women with endometriosis who were treated with an intrauterine contraceptive device containing levonorgestrel. Fertil Steril, 83:398-404.

Luukkainen T, Lahteenmaki P, Toivonen J. 1990. Levonorgestrel-releasing intrauterine device. Ann Med, 22:85-90.

Marjoribanks J, Lethaby A, Farquhar C. 2006. Surgery versus medical therapy for heavy menstrual bleeding. Cochrane Database Syst Rev, 19(2):CD003855.

Mohllajee AP, Curtis KM, Peterson HB. 2006. Does insertion and use of an intrauterine device increase the risk of pelvic inflammatory disease among women with sexually transmitted infection? A systematic review. Contraception, 73:145-53.

[NICE] National Institute for Health and Clinical Excellence Guidelines. 2005. Long acting reversible contraception.

Nilsson CG, Lahteenmaki P, Luukkainen T. 1980. Levonorgestrel plasma concentrations and hormone profiles after insertion and after one year of treatment with a levonorgestrel-IUD. Contraception, 21:225-33.

Odlind V. 1996. Modern intrauterine devices. Baillieres Clin Obstet Gynaecol, 10:55-67.

Pakarinen P, Toivonen J, Luukkainen T. 2003. Randomized comparison of levonorgestrel- and copper-releasing intrauterine systems immediately after abortion, with 5 years' follow-up. Contraception, 68:31-4.

Petta CA, Ferriani RA, Abra o MS, et al. 2005. Randomized clinical trial of a levonorgestrel-releasing intrauterine system and a depot $\mathrm{GnRH}$ analogue for the treatment of chronic pelvic pain in women with endometriosis. Hum Reprod, 20:1993-8.

Pisoni CN, Cuadrado MJ, Khamashta MA, et al. 2005. Treatment of menorrhagia associated with oral anticoagulation: efficacy of levonorgestrel releasing intrauterine device. Thromb Res, 115(Suppl 1):121-2.

Prager S, Darney PD. 2007. The levonorgestrel intrauterine system in nulliparous women. Contraception, 75(6 Suppl):S12-5. 
Progress in Reproductive Health Research, Issue 60, WHO 2002.

Riphagen FE. 2000. Intrauterine application of progestins in hormone replacement therapy: a review. Climacteric, 3:199-211.

Rönnerdag M, Odlind V. 1999. Health effects of long-term use of the intrauterine levonorgestrel-releasing system. A follow-up study over 12 years of continuous use. Acta Obstet Gynecol Scand, 78:716-21.

Shaamash AH, Sayed G, Hussien M, et al. 2005. A comparative study of the levonorgestrel-releasing intrauterine system Mirena versus the Copper T380A intrauterine device during lactation: breast-feeding performance, infant growth and infant development. Contraception, 72:346-51.

Sitruk-Ware R. 2007. The levonorgestrel intrauterine system for use in periand postmenopausal women. Contraception, 75:S155-S60.

Sivin I, Stern J, Coutinho E, et al. 1991. Prolonged intrauterine contraception: a seven-year randomized study of the levonorgestrel $20 \mathrm{mcg} /$ day (LNg 20) and the Copper T380 Ag IUDS. Contraception, 44:473-80.

Soysal S, Soysal ME. 2005. The efficacy of levonorgestrel-releasing intrauterine device in selected cases of myoma-related menorrhagia: a prospective controlled trial. Gynecol Obstet Invest, 59:29-35.

Suhonen S, Haukkamaa M, Jakobsson T, et al. 2004. Clinical performance of a levonorgestrel-releasing intrauterine system and oral contraceptives in young nulliparous women: a comparative study. Contraception, 69:407-12.

Thonneau PF, Almont TE. 2008. Contraceptive efficacy of intrauterine devices. Am J Obstet Gynecol, 198:248-53.

Toivonen J, Luukkainen T, Allonen H. 1991. Protective effect of intrauterine release of levonorgestrel on pelvic infection: three years ' comparative experience of levonorgestrel- and copper-releasing intrauterine devices. Obstet Gynecol, 77:261-4.

Trinh XB, Tjalma WA, Makar AP, et al. 2008. Use of the levonorgestrelreleasing intrauterine system in breast cancer patients. Fertil Steril, 90:17-22.

Varma R, Sinha D, Gupta JK. 2006. Non-contraceptive uses of levonorgestrel-releasing hormone system (LNG-IUS) - a systematic enquiry and overview. Eur J Obstet Gynecol Reprod Biol, 125:9-28.
Varma R, Soneja H, Bhatia K, et al. 2008. The effectiveness of a levonorgestrel-releasing intrauterine system (LNG-IUS) in the treatment of endometrial hyperplasia-A long-term follow-up study. Eur J Obstet Gynecol Reprod Biol, Apr 25 [Epub ahead of print].

Van Houdenhoven K, van Kaam KJAF, van Grootheest AC, et al. 2006. Uterine perforation in women using a levonorgestrel-releasing intrauterine system. Contraception, 73:257-60.

Vercellini P, Frontino G, De Giorgi O, et al. 2003. Comparison of a levonorgestrel-releasing intrauterine device versus expectant management after conservative surgery for symptomatic endometriosis: a pilot study. Fertil Steril, 80:305-9.

WHO. 2004. Medical eligibility criteria for contraceptive use. World Health Organization. URL: http://www.who.int/reproductive-health/ publications/mec/7_iud.pdf

World Health Organization Department of Reproductive Health and Research (WHO/RHR) and Johns Hopkins Bloomberg School of Public Health/Center for Communication Programs (CCP), INFO Project. 2007. Family Planning: A Global Handbook for Providers. Baltimore and Geneva: CCP and WHO.

Wildemeersch D. 2007. New frameless and framed intrauterine devices and systems - an overview. Contraception, 75(6 Suppl):S82-92.

Wildemeersch D, Janssens D, Pylyser K, et al. 2007b. Management of patients with non-atypical and atypical endometrial hyperplasia with a levonorgestrel-releasing intrauterine system: long-term follow-up. Maturitas, 57: 210-3.

Wildemeersch D, Pylyser K, Wever N, et al. 2007a. Endometrial safety after 5 years of continuous combined transdermal estrogen and intrauterine levonorgestrel delivery for postmenopausal hormone substitution. Maturitas, 57:205-9.

Zalel Y, Gamzu R, Shulman A, et al. 2003. The progestative effect of the levonorgestrel-releasing intrauterine system - when does it manifest? Contraception, 67:473-6.

Xiao B, Wu SC, Chong J, et al. 2003. Therapeutic effects of the levonorgestrel-releasing intrauterine system in the treatment of idiopathic menorrhagia. Fertil Steril, 79:963-9. 
